EPJ Web of Conferences 19, 07003 (2012)

DOI: $10.1051 /$ epjconf/20121907003

(C) Owned by the authors, published by EDP Sciences, 2012

\title{
Mechanisms based on chaotic flows that support structures in barred-spiral systems
}

\author{
P.A. Patsis ${ }^{a}$ \\ Research Center for Astronomy, Academy of Athens, Soranou Efessiou 4, \\ 11527 Athens, Grece
}

\begin{abstract}
I summarize the dynamical mechanisms that have been found to shape structures such as the spirals and the bars in barred-spiral systems. Response models and orbital analysis are used to study the dynamics in potentials estimated from near-infrared images of galaxies and are considered therefore as realistic. Contrarily to what is found in normal (non-barred) spirals, where the spiral arms are supported by regular orbits trapped around stable periodic orbits, in barred spiral systems the spirals are reinforced by particles in chaotic motion. The standard mechanism is associated with the unstable Lagrangian points close to the ends of the bar. Then the principal, "chaotic", spirals extend beyond corotation. However, there are also cases where the chaotic orbits that support the spirals stay always inside corotation. Particles in chaotic motion are found to reinforce also the outer envelopes of the bars.
\end{abstract}

\section{INTRODUCTION}

In the last years it has been realized that the spirals emerging out of the ends of the bars can be supported by stars in chaotic orbits. Since 2005, there are more than 33 papers on the subject in journals and conference proceedings. An almost complete list of these papers can be found in the References of the present paper from [1] to [33]. The main mechanism proposed is associated with the unstable manifolds of the orbits of the unstable family of periodic orbits generated at the Lagrangian points $\mathrm{L}_{1}$ and $\mathrm{L}_{2}$ near the ends of the bar. However, alternative mechanisms have been also proposed (see [29]). The role of chaotic orbits in supporting the spirals in barred-spiral galaxies has been underlined earlier (1996) by Kaufmann and Contopoulos ([34]). They proposed that the part of the spirals close to end of the bars is reinforced by chaotic orbits, while the part between the resonances $-4: 1$ and $-2: 1$ by regular orbits. Also in [35] it has been argued that the boxiness of the early type bar of NGC 4314 is due to chaotic orbits. Dynamical mechanisms supporting structures close to the ends of a bar have to be based on stars in chaotic motion, since these structures have to "survive" through the chaotic zone of corotation.

Below we enumerate the structures we found supported by chaotic orbits in potentials estimated from near-infrared images of specific galaxies.

ae-mail: patsis@academyof athens.gr

This is an Open Access article distributed under the terms of the Creative Commons Attribution-Noncommercial License 3.0, which permits unrestricted use, distribution, and reproduction in any noncommercial medium, provided the original work is properly cited. 


\section{THE POTENTIALS}

The potentials of the disks that have been used are based on near-infrared observations of the barredspiral galaxies NGC 4314 ([36]), NGC 1300 ([26]) and NGC 3359 ([37]). They are of the general form:

$$
\Phi(R, \theta)=\Phi_{0}(R)+\sum_{k}^{k_{\max }}\left[\Phi_{k c}(R) \cos (k \theta)+\Phi_{k s}(R) \sin (k \theta)\right] .
$$

Usually $k_{\max }$ up to 6 is enough to describe accurately the morphological features of the galaxy ([26]), while the necessity of including besides the even, also the odd terms depends on the morphology of the specific galaxy. Additional potential terms for a bulge and a dark matter component have been included in the models for NGC 1300. For estimating the amplitudes of the trigonometric terms the thickness of the disks and their M/L are taken into account. The potentials are considered as time-independent, and the bar and the spirals have been assumed to rotate with the same pattern speed. Equations of motion are derived from the Hamiltonian

$$
H \equiv \frac{1}{2}\left(\dot{x}^{2}+\dot{y}^{2}\right)+\Phi(x, y)-\frac{1}{2} \Omega_{p}^{2}\left(x^{2}+y^{2}\right)=E_{J},
$$

where $(x, y)$ are the coordinates in the Cartesian frame of reference, rotating with angular velocity $\Omega_{p}$. $\Phi(x, y)$ is the potential in Cartesian coordinates. $E_{J}$ is the numerical value of the Jacobi constant and dots denote time derivatives.

The results we present here are independent of the best set of parameters found to describe in the most accurate way the photometry and the kinematics of the galaxies. We refer to structures obtained in response models for these potentials as we vary the pattern speed $\Omega_{p}$.

\section{3. "CHAOTIC" STRUCTURES}

\subsection{Bars supported by chaotic orbits}

The part of the bars that has been found to be reinforced by chaotic orbits is an outer envelope of them. This means that we always find a central bar region supported in the known way by regular orbits trapped around stable $\mathrm{x}_{1}$ periodic orbits, surrounded by an envelope that is built by particles in chaotic motion. Usually, but not always, the thickness of this envelope is small and corresponds to the outer isophotes of the bar of the galaxy. In particular:

- In the case of the potential of NGC 4314, under the assumption that $\mathrm{L}_{1}$ and $\mathrm{L}_{2}$ are near the ends of the bar, we find that the shape of the bar is due to chaotic orbits $([3,32,35])$. The orbits of the particles that support the outer boxy isophotes of this galaxy ([36]) are in chaotic motion.

- In a model studying the dynamics of NGC 1300 (with $\Omega_{p}=23 \mathrm{~km} / \mathrm{s} / \mathrm{kpc}$ ) the ansae character of the bar was due to the chaotic motion of the particles in an effective potential with multiple Lagrangian points roughly along the major axis of the bar ("Model 1" in [29]). In that case the role of chaos was more pronounced, in the sense that the regular orbits were building only the central region of the model bar.

- In both cases above orbits building the outer envelopes of the bars were also supporting the spirals emerging out of the ends of the bars ([3], [29], [32]). Such behavior has been also found in $N$-body models ([5]).

\subsection{Spirals supported by chaotic orbits}

- "Chaotic" spirals emerging out of the ends of the bar are found in all cases in which we populate the region of the unstable Lagrangian points $\mathrm{L}_{1}$ and $\mathrm{L}_{2}$. These stellar spirals faint as we move azimuthally along them, away from the ends of the bar. In the NGC 4314 models ([3]) the azimuthal extent of 


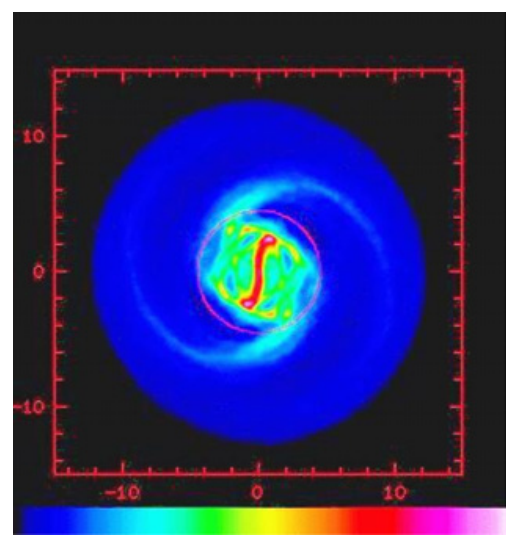

Figure 1. A stellar model that combines ordered and chaotic flows. The circle represents corotation. The bar inside corotation is built by particles in regular orbits, while the spirals beyond corotation are "chaotic" (axes in kpc).

the spirals was less than $\pi / 2$. The velocity dispersion in the initial conditions of the response models plays a role as regards the azimuthal extent of the chaotic spirals. Colder initial conditions favor larger azimuthal extents. Especially in gaseous models the drift of SPH particles along the unstable manifold of the unstable periodic orbits associated with $\mathrm{L}_{1}, \mathrm{~L}_{2}$, leads to extended spiral arms ([23]).

- In another model of the NGC 1300 potential (with $\Omega_{p}=16 \mathrm{~km} / \mathrm{s} / \mathrm{kpc}$, "Model 2" in [29]) we have found spirals supported by chaotic orbits inside corotation. The Jacobi constants of the particles supporting these spirals are close to but smaller than the Jacobi constants of $\mathrm{L}_{1}$ and $\mathrm{L}_{2}\left(E_{L}\right)$. These orbits are sticky orbits having $E_{J}$ 's corresponding to the regions of $n: 1$ resonances with $n \gtrsim 8$.

\section{DISCUSSION}

In all cases with "chaotic" spirals we have studied, the spiral arms have their origin in the corotation region close to the ends of the bar. Even in the case in which the particles supporting the spirals have $E_{J}<E_{L}$, the whole spiral structure extends within a narrow ring just inside corotation. The orbital dynamics that support the spirals in barred-spiral systems are different from the one proposed by Contopoulos and Grosbøl ([38]) for the case of normal (non-barred or weakly barred) spirals. In the latter case the strong symmetric spirals extend between the $2: 1$ and $4: 1$ resonances, while the Lagrangian points are at the end of the overall spiral structure (strong spirals plus weak and asymmetric extensions) that we observe in a galaxy of this morphological type. Then, if the main spiral extends within 2-3 exponential scale lengths of the galactic disk, the Lagrangian points will be located in a region depleted from stars and at radii where the perturbing forces will be a small fraction of the axisymmetric ones. Thus, the dynamical mechanism supporting the formation of "chaotic" spirals will be difficult to act. In barred-spiral systems ordered flows can be observed only in cases where the dynamics of the spirals is to a large extent decoupled from that of the bar, e.g. in cases where the two components have different pattern speeds. Such a case was encountered in the potential of NGC 3359 ([37]). In a barredspiral model with a bar having a maximum relative force perturbation about $40 \%$ of the axisymmetric background, and close to the end of the bar about $20 \%$, the spirals of the model that were matching best the spirals of the galaxy were formed in a regular "precessing ellipses" flow. In order to combine these spirals with a bar ending close to its corotation one had to assume different pattern speeds for the bar and the spirals.

Fig. 1 shows a model in a modified NGC 3359 potential ([37]) rotating with $30 \mathrm{~km} / \mathrm{s} / \mathrm{kpc}$ that shows an ordered flow inside corotation (drawn circle) and chaotic spirals beyond it (Tsigaridi and Patsis in preparation). 
EPJ Web of Conferences

This work has been partially supported by the Research Committee of the Academy of Athens through the project 200/739.

\section{References}

[1] Voglis, N., Stavropoulos I., in "Recent advances in Astronomy and Astrophysics", (N. Solomos ed, AIP Conference Proceedings, Volume 848, Kefalonia, Greece, 2005), 647-659

[2] Voglis, N., in Proceedings of Bernard's Cosmic Stories: from primordial fluctuations to the birth of stars and galaxies (UIMP, VALENCIA, SPAIN, 2006), 60.1

[3] Patsis, P.A., MNRAS 369L (2006) L56-L60

[4] Romero-Gómez, M., Masdemont J. J., Athanassoula E., García-Gómez C. A\&A 453 (2006) 39-45

[5] Voglis N., Stavropoulos I., Kalapotharakos, C., MNRAS 372, (2006) 901-922

[6] Voglis, N., Tsoutsis P., Efthymiopoulos C., MNRAS 373 (2006) 280-294

[7] Athanassoula, L., Romero-Gomez M., Masdemont J., Garcia, C. in "Dynamics of Galaxies", (Pulkovo Observatory, St. Petersburg, Russia, 2007) 1

[8] Romero-Gómez, M., Athanassoula E., Masdemont J. J., García-Gómez, C., A\&A 472 (2007) 63-75

[9] Contopoulos, G., in Chaos in Astronomy (Astrophysics and Space Science Proceedings, G. Contopoulos and P.A. Patsis eds., Springer-Verlag Berlin Heidelberg, Athens, Greece, 2007) 3-22

[10] Efthymiopoulos, C., Tsoutsis P., Kalapotharakos C., Contopoulos G., in Chaos in Astronomy (Astrophysics and Space Science Proceedings, G. Contopoulos and P.A. Patsis eds., SpringerVerlag Berlin Heidelberg, Athens, Greece, 2007) 173-182

[11] Patsis, P.A., in Chaos in Astronomy (Astrophysics and Space Science Proceedings, G. Contopoulos and P.A. Patsis eds., Springer-Verlag Berlin Heidelberg, Athens, Greece, 2007) 33-44

[12] Romero-Gómez, M., Athanassoula E., Masdemont J. J., García-Gómez, C., Chaos in Astronomy (Astrophysics and Space Science Proceedings, G. Contopoulos and P.A. Patsis eds., SpringerVerlag Berlin Heidelberg, Athens, Greece, 2007) 85-92

[13] Tsoutsis, P., Efthymiopoulos C., in Chaos in Astronomy (Astrophysics and Space Science Proceedings, G. Contopoulos and P.A. Patsis eds., Springer-Verlag Berlin Heidelberg, Athens, Greece, 2007) 247-251

[14] Voglis, N., Harsoula M., Contopoulos G., MNRAS 381 (2007), 757-770

[15] Romero-Gómez, M., in Semaine de l'Astrophysique Francaise (J. Bouvier, A. Chalabaev, C. Charbonnel eds, Grenoble, France 2007)

[16] Tsoutsis, P., Efthymiopoulos C., Voglis N., MNRAS 387, (2008) 1264-1280

[17] Athanassoula, E., Romero-Gómez M., Masdemont J. J., Tumbling, "Twisting, and Winding Galaxies: Pattern Speeds along the Hubble Sequence”, (E. M. Corsini and V. P. Debattista eds., Padova, Italy, 2008) (MeMSAIt 18, 97)

[18] Patsis, P.A., Kalapotharakos C., in "Twisting, and Winding Galaxies: Pattern Speeds along the Hubble Sequence”, (E. M. Corsini and V. P. Debattista eds., Padova, Italy, 2008) (MeMSAIt 18, 83)

[19] Athanassoula, E., Romero-Gómez M., Masdemont J. J., MNRAS 394, (2009) 67-81

[20] Harsoula, M., Kalapotharakos C., MNRAS 394 (2009) 1605-1619

[21] Tsoutsis, P., Kalapotharakos C., Efthymiopoulos C., Contopoulos G., A\&A (2009) 495, 743-758

[22] Harsoula, M., Kalapotharakos C., Contopoulos G., in Proceedings of the 9th International Conference of the Hellenic Astronomical Society (K. Tsinganos, D. Hatzidimitriou, T. Matsakos eds. ASP Conf. Ser. 424, Athens, Greece, 2009) 377-379 
[23] Tsigaridi, L., Patsis P.A., in Proceedings of the 9th International Conference of the Hellenic Astronomical Society K. Tsinganos, D. Hatzidimitriou, T. Matsakos eds. ASP Conf. Ser. 424, Athens, Greece, 2009) 382-383

[24] Romero-Gómez, M., Masdemont, J. J., García-Gómez, C., Athanassoula, E., Comm. Nonlin. Sc. Num. Sim. 14, (2009) 4123-4138.

[25] Athanassoula, E., Romero-Gómez M., Bosma A., Masdemont J. J., MNRAS 400 (2009) 1706-1720

[26] Kalapotharakos, C., Patsis P.A., Grosbøl P. MNRAS 403, (2010a) 83-95

[27] Athanassoula, E., Romero-Gómez M., Bosma A., Masdemont J. J., MNRAS 407 (2010) 1433-1448

[28] Kalapotharakos, C., Patsis P.A., Grosbøl P., MNRAS 408, (2010b) 9-21

[29] Patsis, P.A., Kalapotharakos C., Grosbøl P., MNRAS 408, (2010a) 22-39

[30] Patsis, P.A., Kalapotharakos C., Grosbøl P., MNRAS 409, (2010b) L94-L98

[31] Harsoula, M., Kalapotharakos C., Contopoulos G., MNRAS 411, (2011) 1111-1126

[32] Chatzopoulos, S., Patsis, P.A., Boily, C. M., MNRAS 416 (2011) 479-492

[33] Romero-Gómez, M., Athanassoula E., Antoja T., Figueras, F., MNRAS tmp.1587 (2011)

[34] Kaufmann, D.E., Contopoulos G., A\&A, 309, (1996) 381-402

[35] Patsis, P.A., Athanassoula E., Quillen A. C., ApJ 483 (1997) 731-744

[36] Quillen, A.C., Frogel J.A., Gonzalez R.A., ApJ 437 (1994), 162-172

[37] Patsis, P.A., Kaufmann D.E., Gottesman S.T., Boonyasayt V., MNRAS 394 (2009) 142-156

[38] Contopoulos, G. \& Grosbøl P., A\&A, 155, (1986), 11-23 